\title{
BIOQ-BYWORD
}

Journal of Clinical and Nursing Research

\section{Study on the Clinical Application of Pain Treatment Efficacy Evaluation and the Nursing of Patients with Chronic Non-cancer Pain}

Qian Han

\section{ARTICLE INFO}

Article history:

Published online: $31^{\text {st }}$ Mar, 2018

Key words:

Evaluation model

Patients with chronic non-cancer pain

Pain

\begin{abstract}
Objective: To evaluate the pain degree of the patients with chronic non-cancer pain by using the evaluation model constituted by heart rate variability, anxiety and depression scale and quality of life rating scale. This study also aims toevaluate the efficacy after treatment and nursing intervention. Methods: 100 patients with chronic non-cancer pain treated in the hospital from February 2016 to April 2017 were selected to compare their heart rate variability, score of anxiety and depression, score of quality of life and NRS score before and after treatment and nursing intervention. Results: After treatment and nursing intervention, the heart rate variability time domain SDNN significantly increased $(\mathrm{P}<0.05)$. The score of anxiety and depression was lower than that before intervention $(\mathrm{P}<0.05)$ while the scores of various dimensions of quality of life were higher than those before intervention $(\mathrm{P}<0.05)$. The results also showed that NRS score was lower than that before intervention $(\mathrm{P}<0.05)$. Conclusion: These findings suggest that the measurement combination of heart rate variability, anxiety and depression scale and quality of life rating scale can be used as an evaluation model to evaluate the pain degree of the patients with chronic non-cancer pain the efficacy after treatment and nursing intervention, which is worthy of clinic application.
\end{abstract}

\section{Introduction}

Pain is a kind of painful experience in sensory, emotional, cognitive and social dimensions related to tissue damages or potential tissue damages ${ }^{[1]}$, which can be clinically divided into acute pain and chronic pain. Among which, chronic pain refers to continuous or intermittent pain for 3 months or over ${ }^{[2]}$. Due to long duration and lack of attention, most of the patients suffering from chronic pain are not treated effectively. They often suffer from the pain silently and are tortured physically and mentally for a long time, causing severe depression and anxiety, which affect the patients' quality of life and bring great loss to the society ${ }^{[3]}$. With the changing of people's living concept, unhealthy phenomena caused by symptoms of pain have gradually attracted the attention of the medical field ${ }^{[4]}$.

At present, the incidence of chronic pain in the general 
population in China is increasing every year ${ }^{[5]}$. This phenomenon has attracted much attention on the study of chronic pain. However, there is still no a comprehensive and accurate testing system used for judging the grade of pain. In this study, we used an evaluation model constituted by heart rate variability, anxiety and depression scale and quality of life rating scale to evaluate the pain degree of the patients with chronic non-cancer pain and the efficacy after treatment and nursing intervention.

\section{Data and methods}

\subsection{Clinical data}

100 patients (46 male and 54 female patients) with chronic non-cancer pain treated in the hospital from February 2016 to April 2017 were selected as the subjects of the study, with the age ranges from 35 to 78 years old and the average age was $50.4 \pm 1.3$ years old. All the patients participating in this study were well-informed and there was no new and death case before and after the treatment.

\subsection{Inclusion and exclusion criteria}

Inclusion criteria: all kinds of symptoms of chronic noncancer pain caused by strain or degeneration, nerve injury, chronic inflammation, ischemia, blood stasis and emotion. Exclusion criteria: tumor-related patients, such as those suffering from neoplasm invasiveness or accepting antitumor therapy or patients with incomplete data.

\subsection{Treatment and nursing intervention}

1.3.1 Treatment: Doctors developed personalized treatment programs according to patients' conditions and the patients were treated through oral drug administration, nerve block, minimally invasive surgery, etc.

1.3.2 Nursing intervention: Three nursing intervention were taken to the recruited patients. Firstly, the expressions of patients were listened carefully and trust was given: pain is the subjective feeling of patients and has great physiological and psychological efficacy on them, but it has no obvious physical sign. Therefore, the evaluation of pain mainly relies on the patients' chief complaint. Meanwhile, the communication with their families was verified in time. Secondly, comprehensive evaluation: the medical history, degree, medication and the degree of interference in life were well-understood. Finally, dynamic evaluation: (1) the onset of pain, the treatment efficacy and the specific improvement were evalu- ated; (2) the pain changes, efficacies and adverse reactions were monitored closely; (3) the dose of the analgesics was adjusted according to the actual condition of the patients.

When analgesics were given, drug toxicity was minimized under the premise of guaranteeing efficacy to avoid lowering the patients' quality of life. Patients were given timely the information of correct health education, the drug effect, the correct drug administration method and time and the adverse reactions. Besides, psychological nursing, cognitive behavioral therapy for anxiety relaxation, relaxation training and suggestive therapy were provided to the patients, all of which aim to alleviate the pain and enhance analgesic efficacy. Rehabilitation training was combined with and other means to realize both relief of symptoms and reduction of physical and mental damages as well as the improvement of quality of life of the patients.

\subsection{Evaluation methods}

\subsubsection{Heart rate variability (HRV)}

In this study, ZXY-1 HRV detector was adopted by pasting the electrode patch on the arm in a fixed position. Detection and analysis were done when the patients were relaxed. After collecting the pulse or heartbeat signals from 5 minutes of quiet-sitting, the function pointer of the autonomic nervous function was obtained immediately. Heart rate variability adopted the time domain index SDNN (standard deviation of N-N intervals): standard deviation of normal sinus RR intervals. The value of SDNN computed is directly proportionally to $\mathrm{HRV}$, in which the higher the value of SDNN the higher the HRV will be ${ }^{[6]}$.

\subsubsection{Anxiety and depression scale}

The self-rating depression scale (SDS) ${ }^{[7]}: 1$ (no or very little time) -4 (most or total time) four grades were used to express the score. The scores of the 20 items were added to compute the raw total score followed by multiplying by 1.25 to obtain $t$ the integer part, which finally generated the standard total score. According to Chinese norms results, if the standard total score $\geqslant 53$, the patient is suffering from depression. The higher the score is the more serious the depression will be.

The self-rating anxiety scale (SAS) ${ }^{[7]}$ : The scoring method is same as that for SDS. The scores of the 20 items were added to compute the raw total score followed by multiplying by 1.25 to obtain the integer part, which finally generated the standard total score. According to Chinese norms results, if the standard total 
score $\geqslant 50$, the patient is suffering from anxiety. The higher the score is the more serious the anxiety will be.

\subsubsection{SF-36 (the MOS item short from health survey, SF-36) health survey scale}

Quality of life rating scale SF-36 was developed by Institute of Health, New England Medical Center, Boston, USA. [8] In this study, the Chinese version translated by Institute of Social Medicine, Zhejiang University
School of Medicine was used to evaluate 8 aspects of health-related quality of life: physiological function $(\mathrm{PF})$, role physical (RP), body pain (BP), general health $(\mathrm{GH})$, vitality (VT), social function (SF), role emotional (RE) and mental health (MH).

Likert sum method was adopted to add the scores for calculating raw score (Table 1). The transmuted score was then calculated by using the standard formula ${ }^{[8]}$.

Table 1 Calculation of scores of various dimensions used in SF-36 scale

\begin{tabular}{llll}
\hline Dimensions & Actual score of each item & $\begin{array}{l}\text { Possible the lowest score and } \\
\text { possible the highest score }\end{array}$ & $\begin{array}{l}\text { Possible aver- } \\
\text { age score }\end{array}$ \\
\hline Physiological func- & $3 \mathrm{a}+3 \mathrm{~b}+3 \mathrm{c}+3 \mathrm{~d}+3 \mathrm{e}+3 \mathrm{f}+3 \mathrm{~g}+3 \mathrm{~h}+3 \mathrm{i}+$ & 10,30 & 20 \\
tion (PF) & $3 \mathrm{j}$ & 4,8 & 4 \\
Role physical (RP) & $4 \mathrm{a}+4 \mathrm{~b}+4 \mathrm{c}+4 \mathrm{~d}$ & 2,12 & 10 \\
Body pain (BP) & $7+8$ & 5,25 & 20 \\
General health (GH) & $1+11 \mathrm{a}+11 \mathrm{~b}+11 \mathrm{c}+11 \mathrm{~d}$ & 4,24 & 20 \\
Vitality (VT) & $9 \mathrm{a}+9 \mathrm{e}+9 \mathrm{~g}+9 \mathrm{i}$ & 2,10 & 8 \\
Social function (SF) & $6+10$ & 3,6 & 3 \\
Role emotional & $5 \mathrm{a}+5 \mathrm{~b}+5 \mathrm{c}$ & 5,30 & 5 \\
Mental health (MH) & $9 \mathrm{~b}+9 \mathrm{c}+9 \mathrm{~d}+9 \mathrm{f}+9 \mathrm{~h}$ & & \\
\hline
\end{tabular}

\subsubsection{Pain score}

Numeric rating scales (NRS) [9] was used to obtain the pain score. On a 10-points scale, the degree of pain was assessed by patients according to their feeling of pain: 0 means painless; 1-3 means mild pain, which is tolerable and does not affect sleeping; 4-6 means moderate pain, which is intolerable and drug-dependent and has effect on sleeping; 7-10 means severe pain, which is intense and intolerable, analgesics are needed and patients cannot fall asleep and may suffer from nervous disorders and other symptoms.

\subsubsection{Statistical processing}

Statistical analysis was performed by using SPSS 16.0 statistical software. the difference between groups was analyzed by two-tailed Student's t-test. Statistical significance was considered at $\mathrm{P}<0.05$. Data was presented as mean $\bar{x} \pm s$.

\section{Results}

2.1 Comparison of heart rate variability time domain SDNN in the patients before and after treatment and nursing intervention

After treatment and nursing, the value of SDNN was significantly increased $(\mathrm{P}<0.05)$ as shown in Table 2.
Table 2 Comparison of HRV time domain of patients

\begin{tabular}{cc}
\hline Group & SDNN \\
\hline Before interven- & $20.91 \pm 6.71$ \\
tion & \\
After intervention & $28.22 \pm 8.57$ \\
$\mathrm{P}$ & $0.000^{*}$ \\
\hline * indicates $\mathrm{P}<0.05$ &
\end{tabular}

2.2 Improvement of depression, anxiety and other clinical manifestations in patients before and after treatment and nursing intervention

Clinical observation showed that the scores of depression and anxiety after intervention were lower than those before intervention and the result was significantly difference $(\mathrm{P}<0.05)$ as shown in Table 3.

Table 3 Comparison of the scores of depression and anxiety in patients before and after treatment and nursing intervention

\begin{tabular}{ccc}
\hline Group & $\begin{array}{c}\text { Score of depres- } \\
\text { sion }\end{array}$ & Score of anxiety \\
\hline $\begin{array}{c}\text { Before in- } \\
\text { tervention } \\
\begin{array}{c}\text { After in- } \\
\text { tervention }\end{array}\end{array}$ & $58.50 \pm 12.52$ & $50.15 \pm 13.56$ \\
\end{tabular}


$\mathrm{P}$

* indicates $\mathrm{P}<0.05$

\subsection{Comparison of the scores of various dimen- sions of quality of life in patients before and after}

\section{treatment and nursing intervention}

The results showed that the scores of various dimensions of quality of life of patients after treatment and nursing intervention were significantly higher than those before intervention $(\mathrm{P}<0.05)$ as shown in Table 4.

Table 4 Comparison of the scores of various dimensions of quality of life in patients before and after treatment

\begin{tabular}{lccc}
\hline Dimensions & Before intervention & After intervention & $\mathrm{P}$ \\
\hline PF & $51.40 \pm 10.61$ & $78.90 \pm 11.44$ & $0.000^{*}$ \\
RP & $34.75 \pm 10.45$ & $48.75 \pm 12.37$ & $0.000^{*}$ \\
BP & $30.38 \pm 13.45$ & $60.08 \pm 14.85$ & $0.000^{*}$ \\
GH & $39.75 \pm 15.41$ & $64.30 \pm 12.18$ & $0.000^{*}$ \\
VT & $47.65 \pm 11.27$ & $75.90 \pm 12.51$ & $0.000^{*}$ \\
SF & $45.25 \pm 12.69$ & $78.25 \pm 14.55$ & $0.000^{*}$ \\
RE & $42.33 \pm 14.39$ & $66.33 \pm 14.65$ & $0.000^{*}$ \\
MH & $52.44 \pm 15.85$ & $72.04 \pm 14.61$ & $0.000^{*}$ \\
\hline
\end{tabular}

* indicates $\mathrm{P}<0.05$

\subsection{Comparison of NRS scores in patients before and after treatment}

Interestingly, patients given with treatment and nursing intervention had a significant lower NRS $(\mathrm{P}<0.05)$ score compared to normal controls. The results were showed in Table 5.

Table 5 Comparison of NRS scores in patients before and after treatment

\begin{tabular}{cc}
\hline Group & NRS score \\
\hline Before interven- & $6.71 \pm 1.92$ \\
tion & \\
After intervention & $1.80 \pm 1.24$ \\
$\mathrm{P}$ & $0.000^{*}$ \\
\hline
\end{tabular}

* indicates $\mathrm{P}<0.05$

\section{Discussions}

Accumulating evidence showing that certain nursing safety incidents in tumor inpatients have been identified in hospitals. The causes of nursing safety problems in tumor inpatients are analyzed, including several aspects. Firstly, the nursing staff lacks certain legal relevant knowledge. The nurses' cognition of laws and regulations is not enough, and they have insufficient understanding on the relevant legal responsibilities. In the treatment for tumor inpatients, it is possible to have inappropriate language or not timely rescue nursing, which can easily lead to disputes between nurses and patients.
In addition, due to a lack of legal awareness, some nursing staffs do not pay attention to the seriousness and carefulness of nursing records. These actions resulted in the timeliness and rigor of the nursing records are not enough and the relevant records are not very accurate, or may appear the error records, the omission records or the unauthorized alteration situations, all of which have the disadvantageous influence on patient's followup illness concern and the legal evidence ${ }^{[2-3]}$.

Secondly, the nursing safety management of tumor inpatients is not in place. Due to their own emotional and psychological effects as well as the impact of their own diseases, it is very easy for cancer inpatients to having the situations, such as falling or falling out of bed, pressure sore, suicide, lost and other adverse events. The nursing staff cannot judge the related risk factors accurately, and the evaluation of the related activity ability of the patients is insufficient, which causes the improper nursing safety management of the tumor inpatients. There are also individual nursing staff who has judged the nursing risk, but improper implementation of the relevant nursing measures or inadequate implementation still leads to the occurrence of adverse events [4-5].

Thirdly, the implementation of relevant nursing regulations is not enough. When nursing staff provides related nursing work to cancer inpatients, due to relatively heavy nursing tasks or lack of human resources, individual nursing staff may omit some nursing operation steps or check steps or carry out nursing intervention according to their own experience. Moreover, it can also lead to the occurrence of adverse care events in 
hospitalized patients. In addition, the relevant technical operation is not appropriate and succession-related systems as well because the disinfection and isolation steps are not strictly implemented, etc., all of which can easily lead to adverse events of nursing for cancer inpatients ${ }^{[6]}$. Fourthly, the comprehensive quality of nursing staff is deficient. Nursing staff who has relatively weak nursing basic knowledge and operation can easily lead to nursing errors in the process of nursing. Apart from this, individual nursing staff who does not practice high responsibility may result in dispensing errors and needle errors, etc. [7]

Fifthly, poor communication between nurses and patients. Nursing staff who lacks communication skills or nurse-patient communication experience, in which the nurse-patient communication appears back perfunctory, or the answer is relatively blunt and poor attitude, etc. This situation may generate adverse effects on the relationship between nurses and patients and can easily lead to the occurrence of nurse-patient disputes.

Sixthly, the management of medical equipment and articles is not good. The lack of preparation or the poor performance of the related rescue equipment and articles might also affect the rescue of the hospitalized patients. The placement of related items in the ward is relatively disorder, and there are sundries or other conditions, which all can lead to the occurrence of falling or skin damage to the tumor inpatients ${ }^{[8]}$.

Seventhly, there are some factors existed in the patients with tumor. Tumor inpatients have relatively large psychological pressure and prone to have anxiety, depression or other unhealthy psychological diseases. If there is no timely and effective psychological care, there will even be weary, suicidal and other serious adverse events [9-10].

After providing relevant nursing safety management countermeasures to cancer inpatients, the results showed that compared with the calculated values of the tumor inpatients in the reference group, the nursing adverse events of the patients in the experimental group were significantly lower. Similarly, the total rate of nursing disputes in the experimental group was significantly lower than that in the reference group. All data presented were statistically significance at $p<0.05$ data reference and demonstrate the feasibility of coping with nursing safety management in tumor inpatients.

In conclusion, there are some nursing safety problems occurred in hospitalized patients with cancer. The management of nursing safety should be strictly implemented in patients with cancer, which embodies remarkable effect and has the value of popularization.

\section{References}

[1] Williams A C C, Craig K D. Updating the definition of pain [J]. Pain, 2016, 157(11): 2420-2423.

[2] International Pain Summit Of The International Association For The Study Of Pain. Declaration of Montreal: declaration that access to pain management is a fundamental human fight. J Pain Palliat Care Pharmacother. 201 1-25: 29-31

[3] Cui J, Zhou L, Zhang L, et a1. Training the trainer: An educational course for training pain nursing specialists supported by the International Association for the Study of Pain (IASP). Pain Manag Nurs. 2013, 14: e142-150

[4] Li Hua. Adjustment of China's Narcotic Drug Policy and Changes in the Concept of Pain Treatment [J]. Chinese Licensed Pharmacist, 2015, 12(2): 41-45.

[5] Sun Tao. International status of the treatment of interdisciplinary chronic pain. Chinese Journal of Pain Medicine, 2013, 7: 385-386.

[6] Talase B, Kurita A, Noritake M, et al. Heart rate variability in patients with mellitus diabetes, ischemic heart disease and longestive heart failure. J Electrocardiol, 1992, 25(2): 79.

[7] Zhang ZJ. Handbook of behavioral medicine scaes [M/CD]. Beijing: Chinese Medical Multimedia Press, 2005: 213-214, 223-224.

[8] Ware J E, Kosinski M, Dewey J E, et al. SF-36 health survey: manual and interpretation guide [M]. Quality Metric Inc., 2000.

[9] Paice J A, Cohen F L. Validity of a verbally administered numeric rating scale to measure cancer pain intensity [J]. Cancer nursing, 1997, 20(2): 88-93.

[10] Yu Shengyuan, Wang Guochun, Ge Xiaodong, et al. Drug therapy for aged chronic non-cancer pain Consensus of Chinese Experts [J]. Chinese Journal of Pain Medicine, 2016 (5): 321-325. 